\title{
Diagnóstico pré-natal de gêmeos unidos com uso da ressonância nuclear magnética: relato de dois casos
}

\author{
Prenatal diagnosis of conjoined twins by magnetic resonance imaging: report of two cases \\ Alex Sandro Rolland de Souza ${ }^{1,2,3}$, Cynthia Coelho de Medeiros ${ }^{2,3}$, Carlos Noronha Neto ${ }^{2,4}$, \\ Marcelo Marques de Souza Lima ${ }^{2,3,5}$, Gláucia Virgínia de Queiroz Lins ${ }^{2}$
}

\section{RESUMO}

A gemelaridade imperfeita é entidade bastante rara e de grande interesse para fetólogos e obstetras em geral. Sua incidência estimada varia de 1:50.000 a 1:200.000 nascimentos. Seu diagnóstico precoce se faz necessário, tendo em vista sua importância para o prognóstico da gestação, correta determinação da via de parto e o planejamento pós-natal. Os dois casos relatados são de gêmeos unidos diagnosticados no pré-natal através da ultra-sonografia e ressonância nuclear magnética, para estudo do compartilhamento dos órgãos e melhor definição das relações anatômicas. O primeiro par gemelar foi cefalópago, ou seja, unidos pela cabeça, tórax e abdome, com duas pelves e oito membros. O segundo foi toracópago, ou seja, unidos pelo tórax e abdome superior. A ressonância magnética pouco contribuiu para o diagnóstico de gêmeos unidos. Entretanto, se mostrou de grande auxílio na descrição dos órgãos compartilhados entre os fetos, contribuindo na definição do prognóstico fetal.

PALAVRAS-CHAVE: Gêmeos; Gêmeos unidos; Diagnóstico pré-natal; Imagem por ressonância magnética; Ultra-sonografia pré-natal; Relatos de casos [Tipo de publicação]

\section{ABSTRACT}

Conjoined twins have a rare prevalence and special curiosity among physicians and the general population. The reported frequency varies from 1:50,000 to 1:200,000 pregnancies. Its early diagnosis becomes very important when we think about pregnancy management, method of delivery and neonatal care. We describe two cases of conjoined twins diagnosed by ultrasound and magnetic resonance during prenatal care with the aim to better studying the fetus anatomy. The first conjoined twins were cephalopagus sharing head, thorax and abdominal wall and with two pelvis and four arms and four legs. The second were thoracopagus, united by thorax and part of abdomen. Magnetic resonance imaging contribution was not important to diagnose conjoined twins. However, it was useful to describe the shared organs, contributing to define fetal outcome.

KEYWORDS: Twins; Twins, conjoined; Prenatal diagnosis; Magnetic resonance imaging; Ultrasonography, prenatal; Case reports [Publication type]

\footnotetext{
Centro de Atenção à Mulher - Setor de Medicina Fetal do Instituto Materno Infantil Prof. Fernando Figueira - SEMEFE - IMIP - Recife (PE) - Brasil.

1 Mestrando em Saúde Materno-Infantil do Instituto Materno Infantil Prof. Fernando Figueira - IMIP - Recife (PE) - Brasil.

2 Preceptor de Ginecologia e Obstetrícia do Instituto Materno Infantil Prof. Fernando Figueira - IMIP - Recife (PE) - Brasil.

3 Preceptor de Medicina Fetal do Instituto Materno Infantil Prof. Fernando Figueira - IMIP - Recife (PE) - Brasil.

4 Mestrando em Tocoginecologia do Centro Integrado de Saúde Amaury de Medeiros - CISAM - Recife (PE) - Brasil.

5 Mestrando em Obstetrícia pela Universidade Federal de São Paulo - UNIFESP - São Paulo (SP) - Brasil.

Correspondência: Alex Sandro Rolland de Souza

Av. Rui Barbosa, 579, apto 406 - Graças - 52011-040 - Recife - PE - Telefone: (81) 3221-7924 - e-mail: alexrolland@uol.com.br
} 
Introdução

Os gêmeos coligados, que são sempre monozigóticos, são de ocorrência rara na medicina e levam a grande curiosidade por parte da sociedade e também dos profissionais da área de saúde. A incidência dos vários tipos de gêmeos unidos é incerta e poucos indivíduos têm a oportunidade de estudar mais de um caso detalhadamente. De forma geral sua incidência varia entre 1:50.000 e 1:200.000 gestações ${ }^{1-4}$.

Existem vários tipos de gêmeos unidos descritos, sendo o toracópago e o xifópago os mais freqüentes e representam cerca de $75 \%$ dos gêmeos unidos, seguidos dos pigópagos (16\%), isquiópagos $(6 \%)$ e craniópagos $(2 \%)^{5}$. A duplicação incompleta é menos de $1 \%$ e a variedade dipigus é aproximadamente 1:6.000.000 de partos ${ }^{6}$.

Os gêmeos monozigóticos apresentam geralmente incidência constante em todas as raças ${ }^{7}$. Desta forma, a freqüência de gêmeos unidos não é influenciada por fatores raciais, hereditários, idade materna ou paridade ${ }^{6}$. Esses gemelares são sempre simétricos, do mesmo sexo, com partes do corpo iguais e unidos pela mesma estrutura, com exceção do gemelar parasitário, que é assimétrico ${ }^{8}$.

Embora gêmeos monozigóticos sejam em sua maioria do sexo masculino, 70 a $90 \%$ dos gêmeos imperfeitos são femininos ${ }^{3,9}$. Podem estar associados a diversas outras malformações, como gastrointestinais $(33 \%)$ e cardiacas $(25 \%)$, o que piora o prognóstico fetal. Ocorre associação com o polidrâmnio em cerca de $50 \%$ dos casos, em comparação com $10 \%$ das gestações gemelares normais e $2 \%$ das gestações únicas ${ }^{9}$.

Sua etiologia ainda não se encontra esclarecida. Acredita-se que ocorra devido à divisão tardia do disco embrionário, por volta do $13^{\circ}$ ao $15^{\circ}$ dia após a fecundação ${ }^{5,6,8}$. Existem duas teorias, com pontos de vista opostos, para explicar a patogênese dos gêmeos unidos ${ }^{10}$. Segundo a teoria da fusão, ocorre separação completa dos primeiros blastômeros seguida por fusão parcial dos gêmeos monozigóticos ${ }^{8,11}$. Entretanto, já não se acredita que essa teoria seja a base da gemelidade imperfeita ${ }^{8}$. A teoria da fissão sugere a separação incompleta dos primeiros blastômeros, originando os gêmeos unidos, com duplicação apenas daquelas partes que foram completamente separadas ${ }^{11}$.

No passado o diagnóstico pré-natal de gêmeos coligados não era possivel, sendo hoje realizado na maioria dos casos com o uso da ultra-sonografia ${ }^{10,12-18}$. Alguns critérios ultra-sonográficos para diagnóstico foram estabelecidos, como: ausência de membrana divisória, impossibilidade de identificação completa dos corpos fetais durante a exploração ultra-sonográfica, detecção de anomalias fetais e presença de mais de três vasos no cordão umbilical ${ }^{19}$. Atualmente, seu diagnóstico pode ser realizado a partir da $9^{\mathrm{a}}$ semana de gestação ${ }^{17}$, possibilitando a interrupção precoce da gravidez, o que no Brasil ainda não é permitido ${ }^{20}$.

Uma vez estabelecido o diagnóstico é importante determinar o sítio de coligação e o grau de união dos órgãos envolvidos, para que se possa avaliar o prognóstico fetal. Para isto, é necessária avaliação anatômica precisa (inclusive da vascularização) para o adequado planejamento cirúrgico, com o emprego da ressonância magnética e da tomografia computadorizada ${ }^{21}$.

A ultra-sonografia tem sido o método preferencial para o diagnóstico de anomalias congênitas por causa de seu baixo custo, facilidade, acessibilidade e possibilidade de obter imagem em tempo real. Entretanto, no final da gestação, especialmente na presença de obesidade materna ou oligoâmnio, podemos encontrar grandes dificuldades na realização deste exame ${ }^{14}$.

O diagnóstico ultra-sonográfico de gêmeos unidos, atualmente, é relativamente fácil, principalmente no primeiro e segundo trimestres, porém, sua descrição precisa é dificultada. O operador deve estar atento ao estudo do compartilhamento dos órgãos, fato este importante para se determinar o prognóstico fetal, já que apenas aqueles pacientes com órgãos intactos, distintos e com territórios vasculares independentes são passiveis de tratamento cirúrgico pós-natal ${ }^{22}$.

Tais dificuldades podem ser superadas pela ressonância nuclear magnética ${ }^{14}$. Atualmente, essa tecnologia vem ganhando espaço no diagnóstico pré-natal ${ }^{10,18,22,23}$, principalmente devido ao desenvolvimento da técnica ultrafast que permite imagens de alta resolução em curtos intervalos de tempo ${ }^{14}$. Desta forma, não é mais necessária a sedação materna ou fetal para a realização do exame, podendo ser utilizada nos gêmeos unidos para se obterem informações a respeito do compartilhamento dos órgãos fetais.

O presente estudo visa relatar dois casos de gêmeos unidos, bastante raro na literatura destacando a importância do diagnóstico pré-natal pela ultra-sonografia e da ressonância magnética, para avaliação da gravidade dos casos, permitindo assim, um planejamento cirúrgico, quando existir essa possibilidade, e uma assistência obstétrica adequada, seja a conduta expectante ou mesmo a interrupção da gestação.

\section{Relato dos casos}

Todas as informações foram obtidas após assinatura do termo de consentimento livre e esclarecido das pacientes e o estudo foi aprovado pela Comissão de Ética em Pesquisa do Instituto Ma- 


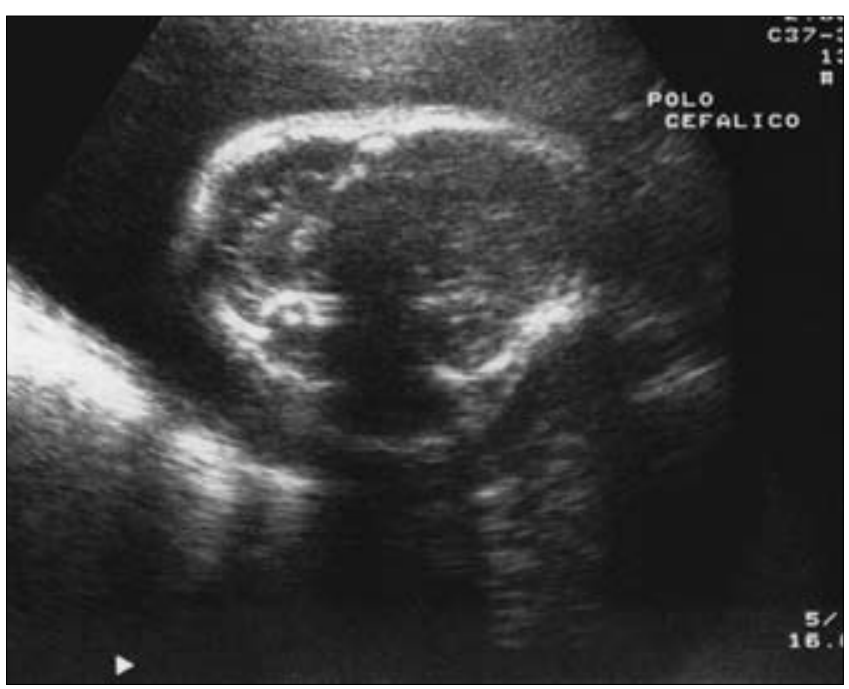

Figura 1 - Imagem ultra-sonográfica do pólo cefálico de gêmeos cefalópagos. Observe a presença de um pólo cefálico de contorno irregular e aumento do diâmetro biparietal com duas órbitas.

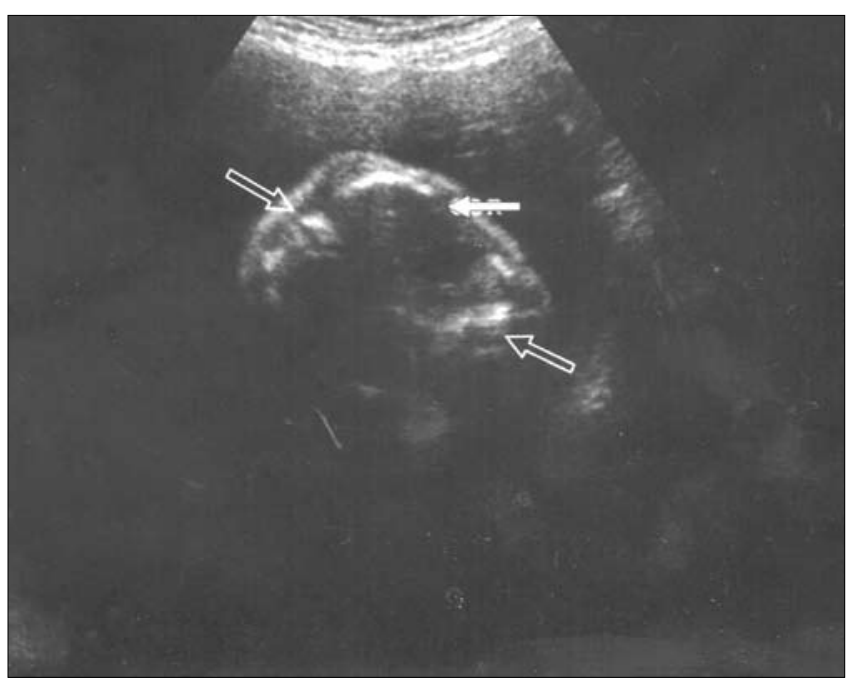

Figura 2 - Imagem ultra-sonográfica em corte transversal do tórax fetal de gêmeos cefalópagos. Observe a presença de duas colunas vertebrais (seta vazada) e a presença de coração único (seta cheia).

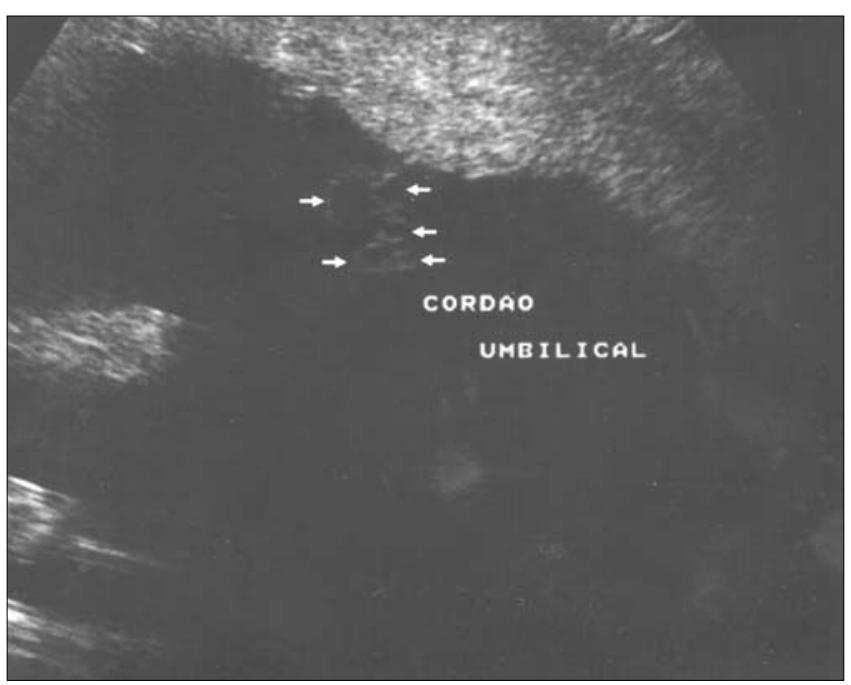

Figura 3 - Imagem ultra-sonográfica em corte transversal do cordão umbilical de gêmeos cefalópagos. Observe a presença de cinco vasos (setas). terno Infantil Professor Fernando Figueira (IMIP), seguindo as orientações do Código de Ética Médica - Resolução do Conselho Federal de Medicina 1246/ 1998 e observados os Princípios Éticos para Pesquisa Médica Envolvendo Indivíduos Humanos descritos na Declaração de Helsinki (1964) e atualizada pela $52^{a}$ Assembléia Geral da Associação Médica Mundial em Edimburgo (2000).

\section{$1^{\circ}$ caso}

R.L.B., 19 anos, I gesta, 0 para, foi admitida no Setor de Medicina Fetal da enfermaria de alto risco do IMIP em 15 de setembro de 2003, com 24 semanas e 5 dias de gestação, encaminhada do pré-natal com suspeita ultra-sonográfica de gemelaridade imperfeita não caracterizada.

Negava doenças prévias à gestação, manobras abortivas, contato com doenças infecciosas, uso de drogas, exposição à radiação e quaisquer outras intercorrências ou antecedentes de gemelaridade. $\mathrm{O}$ exame fisico geral na admissão não detectou anormalidades. Ao exame tocoginecológico houve evidência de candidiase vaginal e condilomatose vulvar, as quais foram adequadamente tratadas. As hipóteses diagnósticas na internação foram de gestação na $24^{\mathrm{a}}$ semana associada a gemelidade imperfeita, polidrâmnio, candidíase vaginal e condilomatose vulvar.

A paciente foi submetida a exames laboratoriais da rotina do pré-natal, tais como, classificação sangüínea, VDRL, HIV, hemograma, sumário de urina e curva glicêmica simplificada, que se encontravam sem alterações.

$\mathrm{Na} 25^{\mathrm{a}}$ semana de gestação foi submetida a exame ultra-sonográfico. No estudo da morfologia fetal, evidenciou-se uma gestação gemelar monocoriônica, monoamniótica, ambos apresentando vitalidade preservada. Notava-se a presença de pólo cefálico único de contorno craniano irregular e com um sistema ventricular cerebral (Figura 1). A face com duas órbitas apresentava hipotelorismo relativo (Figura 1), devido ao aumento do diâmetro biparietal, lábios íntegros, cristalino visualizado e hipognatia. Observou-se a presença de duas colunas vertebrais saindo do pólo cefálico, sem defeitos ou solução de continuidade (Figura 2). O tórax era compartilhado por ambos os fetos, sendo observado coração único de dificil avaliação anatômica pela estática fetal, ritmo cardiaco regular e ecogenicidade pulmonar preservada (Figura 2). O abdome era, também, compartilhado pelos conceptos, com presença de um figado, com alças intestinais de ecogenicidade normal, rins não visualizados devido à estática fetal, tendo sido visualizadas duas bexigas com líquido em seus interiores. Os genitais externos eram compatíveis com o sexo feminino sem anormalidades. Constatou-se a presença de quatro membros superiores 


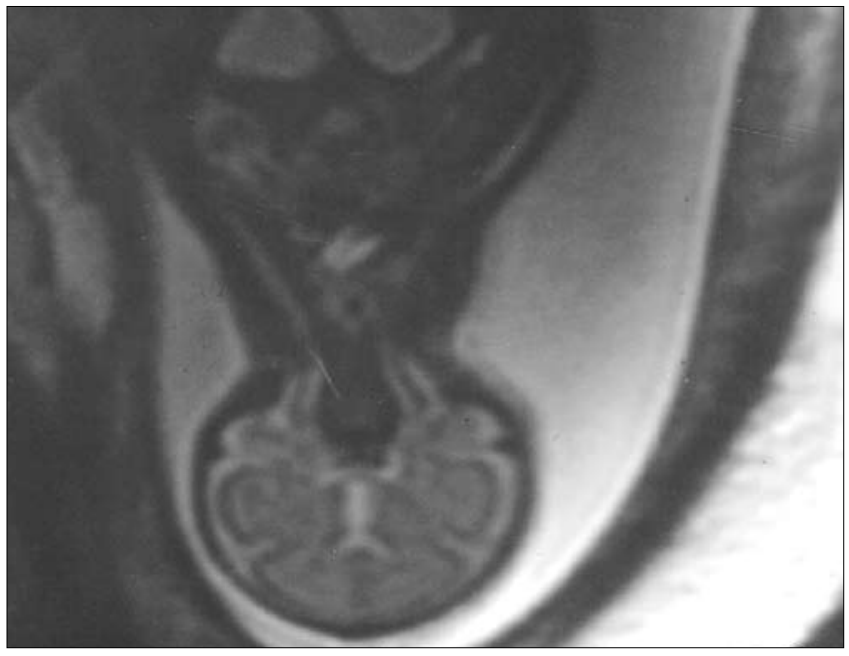

Figura 4 - Imagem de ressonância nuclear magnética em T2 no corte coronal dos gêmeos cefalópagos. Observe a presença de pólo cefálico único e gêmeos unidos pelo tórax e abdome.

e quatro membros inferiores, com mãos e pés bem posicionados. Não havia derrames em cavidades serosas e o cordão umbilical continha cinco vasos (Figura 3). A placenta era de inserção anterior e o índice de líquido amniótico de 38 (aumentado) ${ }^{24}$. O estudo dopplervelocimétrico não detectou alterações nos compartimentos materno, placentário e fetal.

Apesar da dificuldade técnica, devido ao polidrâmnio fetal, a ecocardiografia fetal foi realizada com 25 semanas e 6 dias, sendo visualizado apenas um coração com átrio único e dois ventrículos, dos quais um dominante e outro atrófico.

$\mathrm{Na} 26^{\mathrm{a}}$ semana de gravidez a paciente foi submetida à ressonância nuclear magnética (Figura 4). As seqüências das imagens, em relação ao eixo fetal e com sincronismo respiratório, foram obtidas inicialmente utilizando-se cortes nos planos sagital, axial e coronal gradiente-eco ponderadas em T1. Posteriormente seqüências single-shot fast spin-echo ponderada em T2 foram utilizadas. Observaram-se no sistema nervoso central duas colunas e pólo cefálico único com alterações da calota craniana, predominio do diâmetro látero-lateral, dois hemisférios cerebrais, dois troncos encefálicos unidos no nivel do mesencéfalo e dois cerebelos (Figura 5). As colunas apresentavam-se com dois canais raquidianos contendo duas medulas. A face continha dois globos oculares (Figura 5). O tórax era compartilhado por ambos os fetos, contendo quatro pulmões e um coração com imagem sugestiva de câmara cardíaca única (Figura 6). O abdome apresentava-se com um figado, um estômago, quatros rins e duas bexigas (Figura 7). As extremidades encontravam-se com quatro membros superiores e quatro inferiores e o líquido amniótico estava aumentado.

Aos pais foram esclarecidos os riscos, prognóstico e possibilidades terapêuticas. Diante da

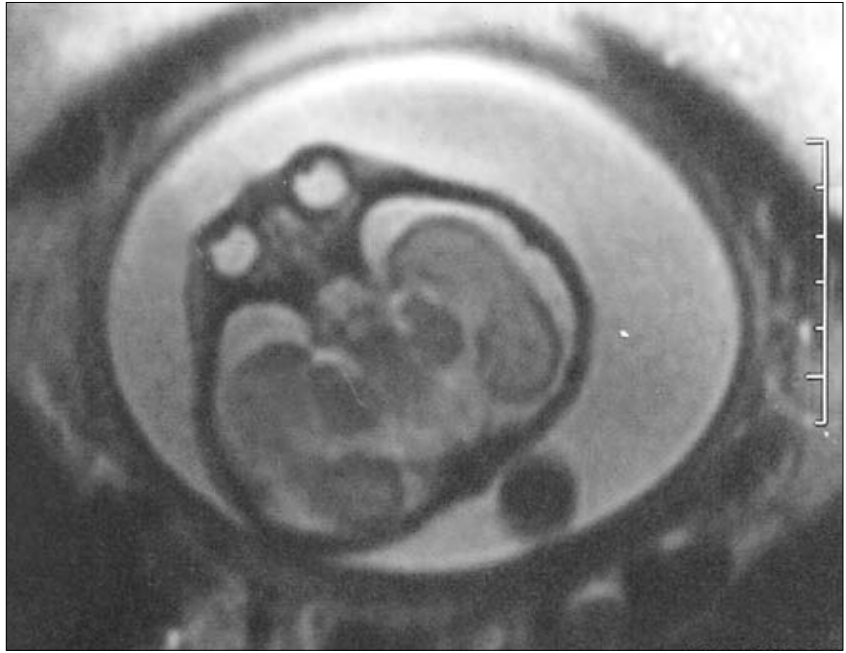

Figura 5 - Imagem de ressonância nuclear magnética em T2 do pólo cefálico de gêmeos cefalópagos. Observe a semelhança com a imagem ultra-sonográfica (Figura 1): presença de duas órbitas, predomínio do diâmetro látero-lateral e dois hemisférios cerebrais.

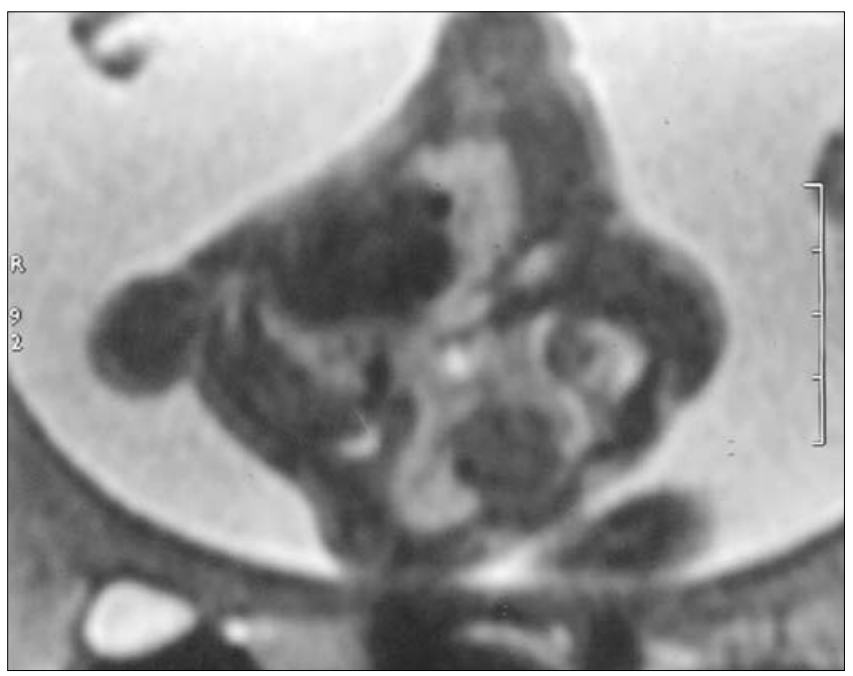

Figura 6 - Imagem de ressonância nuclear magnética em T2 no corte transverso do tórax de gêmeos cefalópagos. Observe a presença de quatro pulmões e coração único.

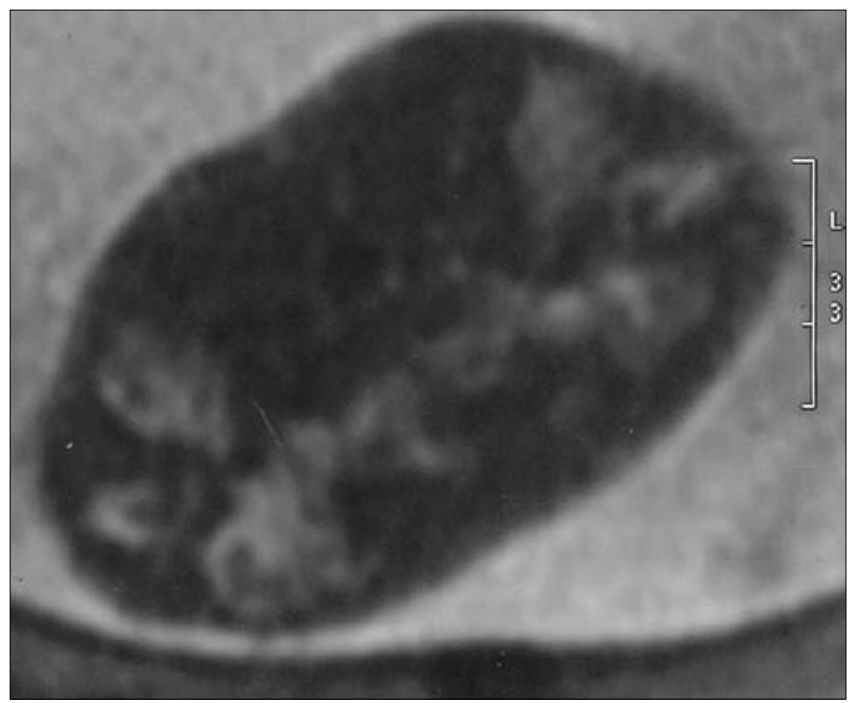

Figura 7 - Imagem de ressonância nuclear magnética em T2 no corte transverso do abdome de gêmeos cefalópagos. Observe a presença de quatro rins. 


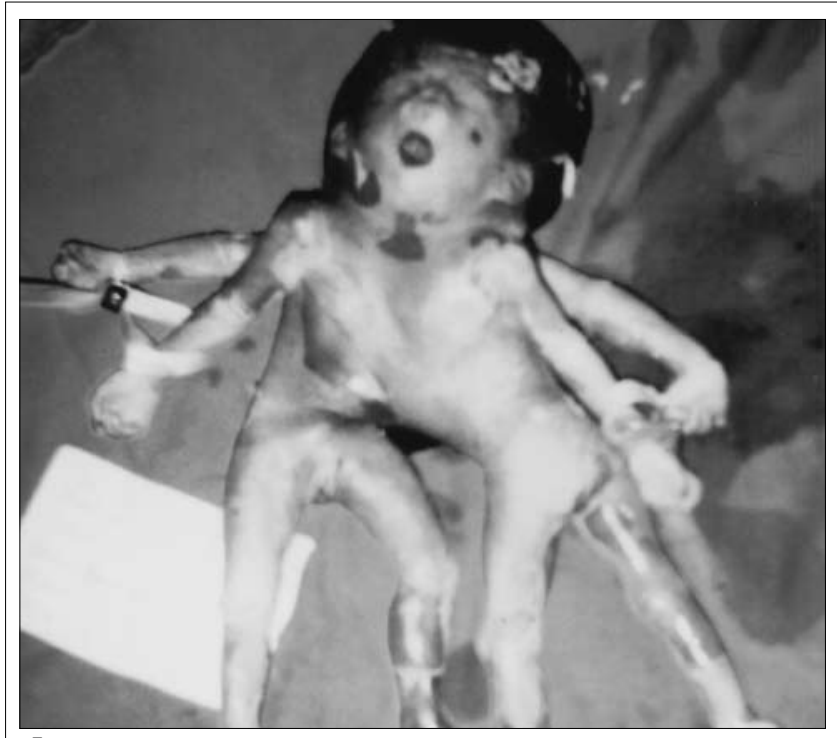

A

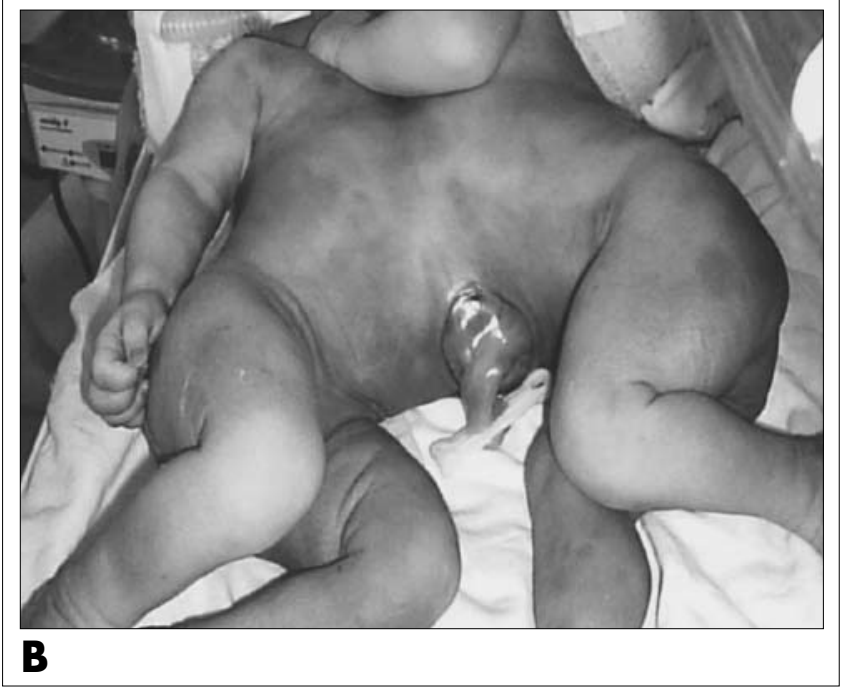

Figura 8 - Aspecto pós-morte dos gêmeos cefalópagos (A) e dos gêmeos toracópagos (B).

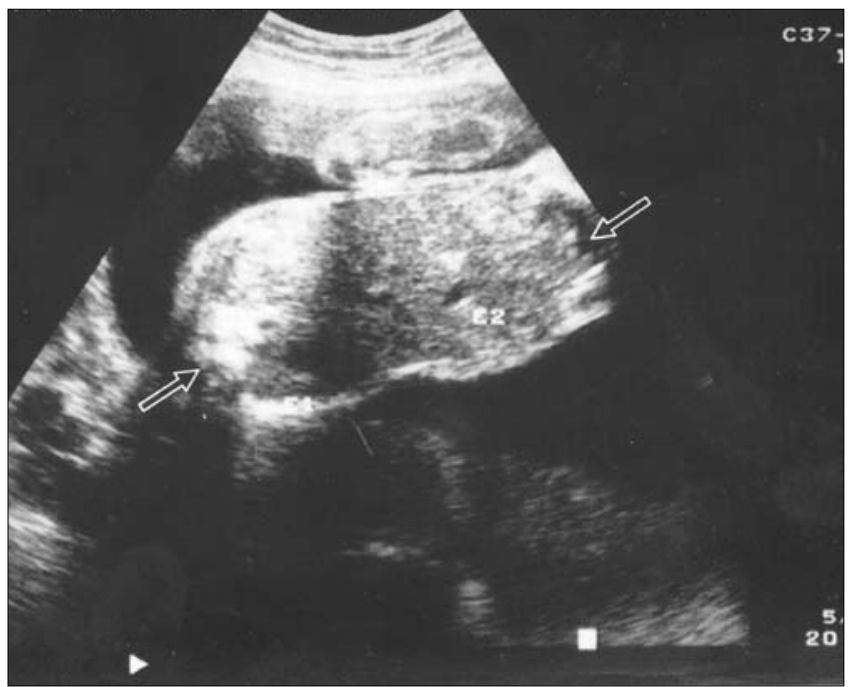

Figura 9 - Imagem ultra-sonográfica em corte transversal do abdome fetal de gêmeos toracópagos. Observe a presença de duas bolhas gástricas $(E)$ e duas colunas vertebrais (setas). restrita chance de sobrevida fetal, da impossibilidade de tratamento cirúrgico pós-natal e do crescente risco materno com o progredir da gestação, além de a paciente expressar o seu desejo pela interrupção, foi solicitada autorização judicial em 3 de outubro de 2003, após relatório médico contendo três ultra-sonografias realizadas por diferentes profissionais, relatório da ressonância magnética, pedido da gestante e pareceres do serviço social e da psicologia. O parecer da justiça foi favorável, sendo iniciada a interrupção médica da gestação em 6 de outubro de 2003 (27 semanas e 5 dias) com misoprostol $50 \mu \mathrm{g}$ de $6 / 6$ horas e posterior indução do trabalho de parto com ocitocina.

Evoluiu para parto transpelviano em 9 de outubro de 2003. Apresentava-se com líquido amniótico aquecido, de odor desagradável e tom achocolatado. Os fetos se apresentavam sem vitalidade, pesando $1.200 \mathrm{~g}$, sexo feminino, sendo confirmadas todas as alterações encontradas (Figuras 8A e 8B).

A paciente foi submetida à curetagem uterina e iniciada antibioticoterapia venosa (clindamicina

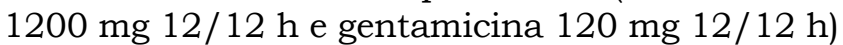
devido à corioamnionite. Recebeu alta hospitalar no quinto dia pós-parto sem outras intercorrências.

\section{$2^{\circ}$ caso}

L.M.S.M., 24 anos, IV gesta, III para, foi admitida no Setor de Medicina Fetal da enfermaria de alto risco do IMIP em 4 de maio de 2005 com 26 semanas e 4 dias, encaminhada do pré-natal com suspeita ultra-sonográfica de gemelidade imperfeita não caracterizada.

Negava doenças prévias à gestação, antecedentes de gemelidade, manobras abortivas, contato com doenças infecciosas, uso de drogas, exposição à radiação e quaisquer outras intercorrências. Os exames físico geral e tocoginecológico não detectaram anormalidades. As hipóteses diagnósticas na internação foram de gestação na $26^{a}$ semana de gravidez associada a gemelidade imperfeita. Os exames laboratoriais da rotina do pré-natal foram normais.

A paciente foi submetida a exame ultra-sonográfico com 26 semanas e 5 dias de gestação. No estudo da morfologia fetal, evidenciou-se gestação gemelar monocoriônica, monoamniótica, ambos apresentando vitalidade preservada. Notava-se a presença de dois fetos coligados através do tórax e porção superior do abdome. Não foi caracterizada a presença de nenhuma malformação associada. O tórax era compartilhado por ambos os fetos, sendo observado coração único, ritmo cardíaco regular e ecogenicidade pulmonar preservada. $\mathrm{O}$ abdome, as alças intestinais e o figado eram compartilhados pelos conceptos (Figura 9). A placenta era de inserção posterior e o índice de líquido 
amniótico encontrava-se normal ${ }^{24}$. O estudo dopplervelocimétrico não apresentou alterações nos compartimentos materno, placentário e fetais. A ecocardiografia fetal foi realizada na $27^{\text {a }}$ semana de gravidez, sendo observado apenas um coração sem alterações anatômicas ou funcionais.

Com 27 semanas e 2 dias de gestação a paciente foi submetida a ressonância nuclear magnética. As seqüências das imagens foram obtidas inicialmente utilizando-se cortes nos três planos gradiente-eco ponderadas em T1. Posteriormente seqüências single-shot fast spin-echo ponderada em T2 foram utilizadas. Observou-se gestação com gêmeos unidos através do tórax e porção superior do abdome, compartilhando-se entre os fetos o coração, figado e alças intestinais. Os dois pólos cefálicos e as duas colunas encontravam-se normais. O tórax apresentava-se com coração único sem malformações e com a presença de quatro pulmões. O abdome encontrava-se com um figado e alças intestinais compartilhadas, além de quatro rins e duas bexigas. Os membros superiores e inferiores foram identificados em número de quatro cada, sem anormalidades (Figuras 10 e 11).

Esta paciente não expressou seu desejo em solicitar a interrupção da gestação e recebeu alta hospitalar com 28 semanas e 4 dias para continuar o pré-natal no ambulatório de medicina fetal, após discussão do caso com a cirurgia pediátrica.

Retornou ao serviço em trabalho de parto na $41^{\text {a }}$ semana de gravidez, sendo submetida a parto cesariano sem intercorrências. Os gêmeos nasceram vivos, do sexo feminino, ambos com indice de Apgar 9 , no primeiro minuto e 10 no segundo minuto, pesando juntos $5.030 \mathrm{~g}$, sendo confirmadas todas as alterações encontradas (Figura 8B). Após quatro horas de vida evoluíram para o óbito. A paciente obteve alta hospitalar após três dias, sem intercorrências.

\section{Discussão}

A avaliação ultra-sonográfica nas gestações é de suma importância para o diagnóstico de uma série de complicações obstétricas, algumas das quais podem influenciar no prognóstico maternofetal. Seu baixo custo e disponibilidade tornam esse exame método complementar indispensável no seguimento obstétrico ${ }^{14}$.

Nas gestações gemelares não poderia ser diferente, sendo a ultra-sonografia utilizada no diagnóstico do número de embriões, da correta idade gestacional, da corionicidade placentária, na avaliação do comprimento da cérvice uterina, para predição do trabalho de parto prematuro, no diagnóstico da sindrome transfusor-transfundido e rastreamento de malformações e aneuploidias, por meio principalmente da translucência nucal ${ }^{7}$, a qual pode estar alterada nos casos de gemelidade imperfeita ${ }^{15}$.

Utilizando uma classificação simplificada ${ }^{25}$, pode-se determinar que no primeiro caso tratase de gêmeos unidos do tipo cefalópagos, que se apresentam com uma cabeça, um tórax e um abdome, porém com duas pelves. Alguns autores podem atribuir nomenclaturas diferentes a esse tipo de gêmeo, como cefalotoracópagos, janiceps, dipagos e outros. O segundo caso é do tipo toracópago, pois os gêmeos se apresentam com dois corpos unidos através do tórax, contendo um

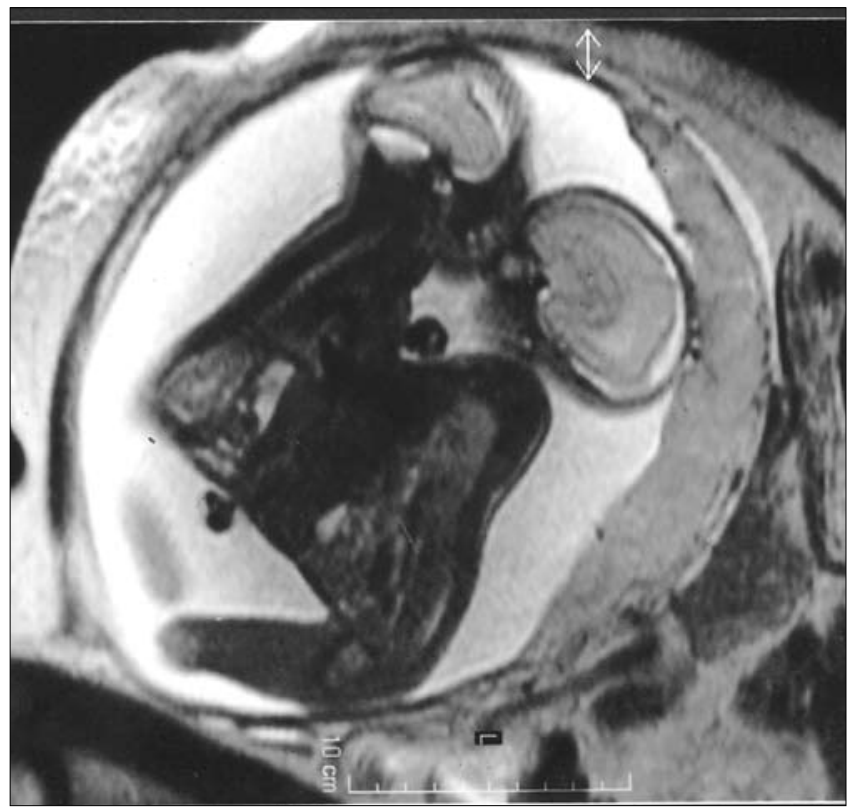

Figura 10 - Imagem de ressonância nuclear magnética em T2 no corte longitudinal dos gêmeos toracópagos.

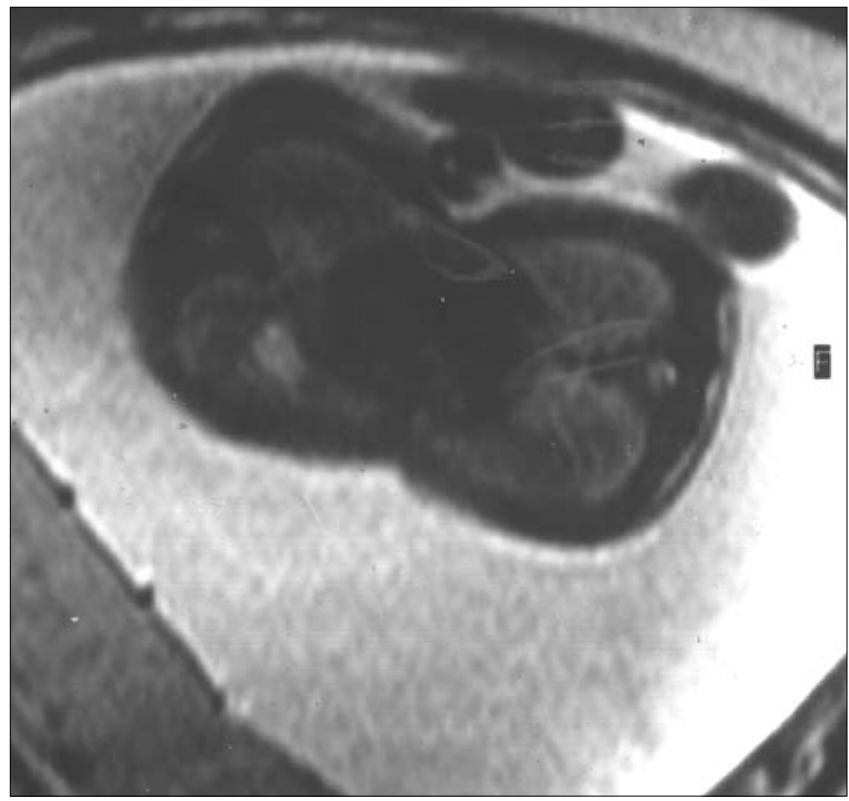

Figura 11 - Imagem de ressonância nuclear magnética em T2 no corte transverso do tórax de gêmeos toracópagos. Observe a presença de quatro pulmões e coração único. 
único coração $0^{3,10,25,26}$. Esse segundo tipo é o mais freqüentemente encontrado na literatura ${ }^{5}$.

De acordo com a literatura, que relata predomínio do sexo feminino e associação com o polidrâmnio e outras malformações congênitas ${ }^{3,5,9}$, ambos os casos eram do sexo feminino. No primeiro caso (cefalópagos) encontramos também a associação com o polidrâmnio e malformação cardíaca, o que agrava o prognóstico fetal.

Nos países desenvolvidos a conduta mais adotada na gemelidade imperfeita é a interrupção da gestação. No Brasil, ou no exterior quando existe a possibilidade de correção cirúrgica, a conduta, geralmente, é expectante ${ }^{1,12}$.

No Brasil, diferentemente de outros países, o abortamento apenas é permitido quando há risco de vida materno e se a gravidez resultar de estupro, devendo ser precedido de consentimento da gestante ou, quando incapaz juridicamente, de seu representante lega ${ }^{20}$. Contudo, os magistrados vêm autorizando judicialmente abortamento em alguns casos de malformações fetais incompativeis com a vida, quando solicitado pela gestante e amparada em relatórios médicos, conforme ocorreu com o primeiro caso.

Segundo os critérios ultra-sonográficos de diagnóstico ${ }^{19}$, observamos a ausência de membrana divisória em ambos os casos, presença de anomalias fetais no primeiro caso e mais de três vasos no cordão umbilical. No cefalópagos foi encontrado cordão umbilical único contendo cinco vasos.

A ressonância nuclear magnética pouco contribuiu para o diagnóstico de gemelidade imperfeita, já que em ambos os casos os diagnósticos já haviam sido realizados com o uso da ultra-sonografia. Entretanto, a determinação de órgãos compartilhados necessita de melhores condições técnicas de imagem, sendo necessários outros métodos complementares para ampliar a sensibilidade da ultra-sonografia, contribuindo para o sucesso do diagnóstico e sendo fundamental para definir o prognóstico fetal ${ }^{23}$. A ressonância nuclear magnética se mostrou de grande auxílio nesta situação, conseguindo confirmar as alterações anatômicas encontradas pela ultra-sonografia e acrescentando ao estudo dos órgãos fetais aqueles que não foram visualizados. Em ambos os casos a ultra-sonografia não conseguiu avaliar os rins e pulmões fetais adequadamente, sendo posteriormente analisados pela ressonância, que identificou a presença de quatro pulmões e quatro rins em cada feto.

A idade gestacional ideal para a sua realização irá depender da doença de base, sendo recomendada após a $24^{a}$ semana de gravidez, periodo após a organogênese. Sua utilização no primeiro trimestre e a utilização de contraste endovenoso não são recomendadas durante a gestação ${ }^{14}$. Nos casos relatados, a ressonância nuclear magnética teve destacada importância ${ }^{18}$ para a confirmação dos órgãos vitais compartilhados, como figado e coração, e conseqüente inviabilidade fetal, com resolução pela interrupção médica da gestação respaldada judicialmente no primeiro caso, a pedido da gestante. Entretanto, a mãe dos gêmeos toracópagos (segundo caso) optou pela conduta conservadora.

$\mathrm{Na}$ atualidade, a literatura cita alguns relatos de casos bem sucedidos após cirurgia corretiva no período pós-natal ${ }^{1}$. Contudo, no primeiro caso foi diagnosticado compartilhamento do pólo cefálico, com dois hemisférios cerebrais (visualizados por meio de ressonância nuclear magnética), duas órbitas, um coração com cardiopatia congênita (diagnosticado por meio da ecocardiografia fetal e ressonância nuclear magnética) e um fígado. Foram considerados gêmeos de prognóstico reservado sem possibilidade cirúrgica no pós-natal. Desta forma, optou-se pela interrupção médica da gestação com o intuito de preservar o futuro reprodutivo materno, já que desta forma o parto por via transpelviana seria factivel de realização nesta idade gestacional ${ }^{27}$.

A maioria dos gêmeos coligados irão a óbito, dos quais cerca de $45 \%$ ainda intra-útero e $25 \%$ nas primeiras 48 horas de vida. Os 30\% restantes apresentam possibilidade de sobrevida com ou sem separação cirúrgica ${ }^{5}$. E daqueles que sobreviverão ao nascimento, aproximadamente $25 \%$ viverão o bastante para serem candidatos à cirurgia ${ }^{8}$. Ambos os casos descritos foram a óbito, sendo o primeiro ainda intra-útero e o segundo após 4 horas de vida.

\section{Referências}

1. Módolo NSP, Amorim RB, Castiglia YMM, Dias SA, Guasti VM. Anestesia para separação de gêmeos isquiópagos no período neonatal: relato de caso. Rev Bras Anestesiol. 2002;52(4):446-52.

2. Shimizu Y, Fukuda J, Sato W, Kumagai J, Hirano H, Tanaka T. First-trimester diagnosis of conjoined twins after in-vitro fertilization-embryo transfer (IVF-ET) at blastocyst stage. Ultrasound Obstet Gynecol. 2004;24(2):208-9.

3. Walker M, Browd S. Craniopagus twins: embryology, classification, surgical anatomy, and separation. Childs Nerv Syst. 2004;20(8-9):554-66.

4. Ossowski KBS, Suskind DL. Airway management in conjoined twins: A rare indication for the EXIT procedure. Arch Otolaryngol Head Neck Surg. 2005;131(1):58-60.

5. Posser AO, Posser ZBR. Gemelaridade imperfeita. Femina. 1996;24(8):739-42. 
6. Ruiz FM, Barrientos RC, Figueroa PJ. Diagnostico antenatal ultrasonografico de gemelos fusionados dipygus. Rev Chil Obstet Ginecol. 1994; 59(4):307-10.

7. Egan JFX, Borgida AF. Multiple gestations: the importance of ultrasound. Obstet Gynecol Clin North Am. 2004;31(1):141-58.

8. Kaufman MH. The embryology of conjoined twins. Childs Nerv Syst. 2004; 20(8-9):508-25.

9. Harper RG, Kenigsberg K, Sia CG, Horn D, Stern D, Bongiovi V. Xiphopagus conjoined twins: A 300-year review of the obstetric, morphopathologic, neonatal, and surgical parameters. Am J Obstet Gynecol. 1980;137(5):617-29.

10.Athanasiadis AP, Tzannatos CT, Mikos T, Zafrakas $\mathrm{M}$, Bontis JN. A unique case of conjoined triplets. Am J Obstet Gynecol. 2005;192(6):2084-7.

11.Botell ML, Fuente AMM, Aguabella ET, Méndez CTP. Siameses: Presentación de un caso. Rev Cubana Med Gen Integr. 1999;15(4):473-5.

12. Costa CFF, Welkovic S, Gurgel FO. Gêmeos acolados: relato de caso. Rev Bras Ginecol Obstet. 1995;17(5):569-71.

13. Chen CP, Shih JC, Shih SL, Huang JK, Haung JP, Lin YH, Wang W. Prenatal diagnosis of cephalothoracopagus janiceps disymmetros using three-dimensional power Doppler ultrasound and magnetic resonance imaging. Ultrasound Obstet Gynecol. 2003;22(3):299-304.

14. Chen PL, Choe KA. Prenatal MRI of heteropagus twins. AJR Am J Roentgenol. 2003;181(6):1676-8.

15.Bulbul Y, Drummond CL, Hillion Y, Bidat L, Ville Y. Diprosopus associated with neural tube defect and facial cleft in the first trimester. Fetal Diagn Ther. 2004;19(3):246-50.

16.Durin L, Hors Y, Jeanne-Pasquier C, Barjot P, Herlicoviez M, Dreyfus M. Prenatal diagnosis of an extremely rare type of conjoined twins: cranio-rachi-pygopagus twins. Fetal Diagn Ther. 2005;20(2):158-60.

17.Fang KH, Wu JL, Yeh GP, Chou PH, Hsu JC, Hsieh CT. Ischiopagus conjoined twins at 9 weeks of gestation: Three-dimensional ultrasound and power Doppler findings. Ultrasound Obstet Gynecol. 2005;25(3):309-10.

18.Iura T, Makinoda S, Sasakura C, Hirosaki N, Inoue $\mathrm{H}$, Waseda T, Watanabe Y. Hemodynamic analysis of cephalothoracopagus by the color Doppler method. A comparison to normal fetuses via a longitudinal study. Fetal Diagn Ther. 2006; 21(1):61-4.

19.Koontz WL, Herbert WN, Seeds JW, Cefalo RC. Ultrasonography in the antepartum diagnosis of conjoined twins. A report of two cases. J Reprod Med. 1983;28(9):627-30.

20.Faúndes A, Duarte GA, Andalaft Neto J, Olivatto AE, Simoneti RM. Conhecimento, opinião e conduta de ginecologistas e obstetras brasileiros sobre o assunto o aborto induzido. Rev Bras Ginecol Obstet. 2004;26(2):89-96.

21.Goodrich J, Staffenberg D. Craniopagus twins: Clinical and surgical management. Childs Nerv Syst. 2004;20(8-9):618-24.

22. Hockley AD, Gornall P, Walsh R, Nishikawa H, Lam H, MacPherson L, et al. Management of pyopagus conjoined twins. Childs Nerv Syst. 2004; 20(8-9):635-9.

23. Salvador D, Ruata MI, Ruiz LD, Travella C, Tinti ME. Diagnostico prenatal de embarazo gemelar dicefálico incompleto. Rev Argent Radiol. 2002;66(3):177-81.

24.Phelan JP, Ahn MO, Smith CV, Rutherford SE, Anderson E. Amniotic fluid index measurements during pregnancy. J Reprod Med. 1987;32(8):601-4.

25.Spencer R. Anatomic description of cojoined twins: a plea for standardized terminology. J Pediatr Surg. 1996; 31(7):941-4.

26.Spitz L, Kiely EM. Conjoined twins. JAMA. 2003;289(10):1307-10.

27.Harma M, Harma M, Mil Z, Oksuzler C. Vaginal delivery of dicephalic parapagus conjoined twins: case report and literature rewiew. Tohoku J Exp Med. 2005;205(2):179-85. 